NOTES

\title{
A NOTE ON THE OPTIMAL CONTROL OF STOCKS ACCUMULATING WITH A DELAY
}

\author{
RALPH WINKLER \\ University of Bern
}

\begin{abstract}
We study a generic optimal control problem with a stock that accumulates with constant delay. We show that the optimal system dynamics reduces to a system of ordinary differential equations, implying monotonic optimal paths, if the objective function is additively separable in the stock and the control. This is, however, not true for general objective functions, which may exhibit nonmonotonic and oscillatory optimal paths. The reason is that the impact of the stock on the objective depends on the current level of the control, whereas the control influences the dynamics of the stock with a delay.
\end{abstract}

Keywords: Additively Separable Objective, Delayed Optimal Control, Differential-Difference Equations, Time to Build

\section{INTRODUCTION}

In many economic problems stocks accumulate with a delay in changes in the control. As an example, one might think of capital investments that do not turn into productive capital instantaneously but exhibit a gestation lag. Such delays already influenced the economic thinking of early authors such as Jevons ([1871]1911) and von Böhm-Bawerk ([1889]1921). Hayek (1941) argued that the time of production is a possible source of aggregate fluctuations, a claim formally derived by Kalecki (1935). This line of argument was revived by the seminal article of Kydland and Prescott (1982), who verified the link between time to build and aggregate fluctuations for U.S. data. Employing delayed differential-difference equations for the capital accumulation equation, Rustichini (1989) and Asea and Zak (1999) showed in simple growth models with one capital good that the investment gestation lag is the driving force of oscillatory system dynamics.

In this paper, we extend this result by analyzing a generic optimal control model with one control that accumulates to a stock with a fixed delay. We show that the

I am grateful to Hans Gersbach and an anonymous reviewer for comments on an earlier draft and to the Simulation and Optimization Group of the Interdisciplinary Center for Scientific Computing, University of Heidelberg for a free license of the MUSCOD-II software package. A precursor to this paper can be found in Winkler (2008). Address correspondence to: Ralph Winkler, Department of Economics and Oeschger Centre for Climate Change Research, University of Bern, Schanzeneckstrasse 1, CH-3001 Bern, Switzerland; e-mail: mail@ralph-winkler.de. 
optimal paths are oscillatory in general but monotonic if the objective is additively separable in the stock and the control. The reason is that for nonadditively separable objective functions the impact of the stock on the objective depends on the current level of the control. The control, however, impacts the stock only with a delay. Under these conditions, nonmonotonic paths may be optimal. As an example, we briefly discuss the optimal control of a long-lived pollutant, the emissions of which accumulate with a delay.

Our result that optimal paths are monotonic in models that employ an additively separable objective has two important implications. First, additively separable objective functions may be a misspecification if they approximate general objective functions, as the functional form influences the qualitative behavior of the optimal paths. Second, the optimal control of delayed stock accumulation does not pose additional analytical complexities compared to instantaneous stock accumulation if the objective function is additively separable.

Our paper complements a recent literature on delayed capital accumulation, which shares two important characteristics. ${ }^{1}$ First, the use of delayed differential equations in optimal control frameworks creates severe analytical difficulties, as the system dynamics is governed by a system of functional differential equations. Second, all contributions exhibit oscillatory optimal paths because of their general equilibrium structure. This implies that the objective is not additively separable with respect to capital (stock) and investments (control), as we explain in the following section.

\section{EXAMPLES}

As the importance of our results crucially depends on whether models exhibit additively separable felicity functions, we start by discussing two popular examples of felicity functions used in economics to clarify what we mean by additive separability.

First, consider the neoclassical growth model with capital as the sole input factor of production. In this general equilibrium model the felicity function $F$ is the instantaneous utility $V(c(t))$ derived from consumption $c$ with the standard properties $V^{\prime}>0, V^{\prime \prime}<0$. Denoting the capital stock of the economy by $k$, production is given by some function $P(k(t))$ satisfying $P^{\prime}>0, P^{\prime \prime}<0$, and Inada conditions. Let us introduce the new control variable investment $i$ with $c(t)=P(k(t))-i(t)$. Then the felicity function $F=V(P(k(t))-i(t))$ is never additively separable in the control $i$ and the stock $k$, due to the general property of decreasing marginal utility $\left(V^{\prime \prime}<0\right)$.

Second, consider the optimal accumulation of a pollution stock in a partial equilibrium framework. Often the objective is to find the net present minimum of the sum of abatement costs $C$, depending on the amount of pollutant emissions $e$, and costs due to environmental damage $D$, depending on the pollutant stock $s .^{2}$ Thus, the felicity function $F=C(e)+D(s)$ is additively separable.

We shall come back to these two examples in Section 4. 


\section{A GENERIC CONTROL MODEL WITH DELAYED STOCK ACCUMULATION}

We introduce a generic optimal control problem with one stock that accumulates with a delay: ${ }^{3}$

$$
\max _{u(t)} \int_{0}^{\infty} F(x(t), u(t)) \exp [-\rho t] d t, \quad \rho>0
$$

subject to

$$
\begin{aligned}
& \dot{x}(t)=u(t-\tau)-\gamma x(t), \quad \tau, \gamma>0, \\
& x(t) \geq 0, \quad u(t) \geq 0, \\
& x(0)=x_{0}, \quad u(t)=\mu(t), \forall t \in[-\tau, 0),
\end{aligned}
$$

where $\rho$ is the constant and positive discount rate. $F$ is a twice continuously differentiable felicity function, which is a function of the stock $x$ and the control $u$. $F$ satisfies Inada conditions and is strictly and jointly concave in both arguments, $x$ and $u$. These assumptions impose the existence of a unique interior solution. To ensure that the control problem converges monotonically toward its unique stationary state in the case of no delay, we further assume that the cross derivative of $F$ is nonpositive [Kamien and Schwartz (1992)].

The dynamics of the stock $x$ is governed by the delayed differential equation (2a), which represents the standard form (except for the delay) used in stock accumulation problems in economics. The stock at time $t$ increases at the level of the control at time $t-\tau$ and deteriorates at the positive and constant rate $\gamma$. Because of this delay $\tau$, the path of the stock $x$ in the time interval $t \in[0, \tau]$ is completely determined by the initial stock $x_{0}$ and the initial control path $\mu$ in the time interval $[-\tau, 0)$. Finally, we impose that both the stock and the control are non-negative at all times.

The maximum principle can be extended to this class of maximization problems [El-Hodiri et al. (1972), Kamien and Schwartz (1992), Kolmanovskii and Myshkis (1999)]. We obtain for the present-value Hamiltonian

$$
\mathcal{H}=F(x(t), u(t)) \exp [-\rho t]+\lambda(t+\tau) u(t)-\gamma \lambda(t) x(t)
$$

where $\lambda$ denotes the costate variable or shadow price of the stock $x$. Assuming that the Hamiltonian $\mathcal{H}$ is continuously differentiable with respect to the control $u$, we obtain the following necessary conditions for an optimal solution:

$$
\begin{gathered}
F_{u}(x(t), u(t)) \exp [-\rho t]=-\lambda(t+\tau), \\
\dot{\lambda}(t)=\gamma \lambda(t)-F_{x}(x(t), u(t)) \exp [-\rho t] .
\end{gathered}
$$


As the Hamiltonian is strictly concave, due to the strict concavity of $F$, these conditions are also sufficient if, in addition, the transversality condition holds:

$$
\lim _{t \rightarrow \infty}[\lambda(t) x(t)]=0
$$

Solving equation (4b) by using the transversality condition (4c) yields

$$
\lambda(t)=\exp [-\rho t] \int_{t}^{\infty} F_{x}\left(x\left(t^{\prime}\right), u\left(t^{\prime}\right)\right) \exp \left[-(\rho+\gamma)\left(t^{\prime}-t\right)\right] d t^{\prime} .
$$

The economic interpretation of the necessary and sufficient conditions is the straightforward generalization of the standard case without delay. The shadow price $\lambda(t)$ equals the net present value of all future (dis)utility stemming from a marginal increase of stock $x$ at time $t$. Equation (4a) says that along the optimal path the net present value of the utility loss (gain) of a marginal increase in the control has to equal the net present value of the utility gain (loss) induced by the resulting increase in the stock. However, the net present value of the utility gain (loss) induced by a marginal increase of the control at time $t$ is equal to $\lambda(t+\tau)$, as the stock accumulates with a timelag $\tau$.

We eliminate the shadow price $\lambda(t)$ by differentiating equation (4a) with respect to time and inserting it into equation (4b). We derive, together with the equation of motion (2a), the following system of differential equations, the solution of which determines the optimal control path $u(t)$ and the optimal stock dynamics $x(t)$ :

$$
\begin{aligned}
& \dot{u}(t)=\frac{1}{F_{u u}(x(t), u(t))}\left\{(\gamma+\rho) F_{u}(x(t), u(t))+F_{x}(x(t+\tau), u(t+\tau)) \exp [-\rho \tau]\right. \\
& \left.+F_{x u}(x(t), u(t))[\gamma x(t)-u(t-\tau)]\right\}, \quad t \geq 0, \\
& \dot{x}(t)=u(t-\tau)-\gamma x(t), \quad t \geq \tau .
\end{aligned}
$$

The optimal stock dynamics $x(t)$ for $t \in[0, \tau]$, which we denote by $\chi(t)$, is given by the initial conditions $(2 \mathrm{c})$ :

$$
\chi(t)=x_{0} \exp [-\gamma t]+\int_{0}^{\tau} \mu\left(t^{\prime}-\tau\right) \exp \left[-\gamma\left(t-t^{\prime}\right)\right] d t^{\prime}, \quad t \in[0, \tau] .
$$

Note that $\dot{u}$ and $\dot{x}$ also depend on advanced (i.e., at a later time) and on retarded (i.e., at an earlier time) variables. In general, this system of functional differential equations exhibits no closed-form analytical solution. Nevertheless, it is possible to derive some qualitative properties of the system dynamics.

We start with the fixed points of the system of functional differential equations (6).

PROPOSITION 1 (Stationary State). The system of functional differential equations (6) exhibits a unique stationary state $\left(x^{\star}, u^{\star}\right)$, which is determined 
by the following system of implicit equations:

$$
\begin{aligned}
-\frac{F_{x}\left(x^{\star}, u^{\star}\right)}{F_{u}\left(x^{\star}, u^{\star}\right)} & =(\gamma+\rho) \exp [\rho \tau], \\
u^{\star} & =\gamma x^{\star}
\end{aligned}
$$

Proof. Set $\dot{x}=\dot{u}=0$, which yields the implicit equations (7). The assumed curvature properties of $F$ together with the Inada conditions imply the existence of a unique solution.

To derive qualitative properties of the system dynamics in a neighborhood around the unique stationary state, we linearize the system of functional differential equations (6) around the stationary state $\left(x^{\star}, u^{\star}\right):^{4}$

$$
\begin{aligned}
\dot{u}(t) & \approx(\gamma+\rho)\left(u(t)-u^{\star}\right)+\frac{F_{x u}^{\star}}{F_{u u}^{\star}} \exp [-\rho \tau]\left(u(t+\tau)-u^{\star}\right) \\
- & \frac{F_{x u}^{\star}}{F_{u u}^{\star}}\left(u(t-\tau)-u^{\star}\right)+\frac{F_{x u}^{\star}}{F_{u u}^{\star}}(2 \gamma+\rho)\left(x(t)-x^{\star}\right) \\
+ & \frac{F_{x x}^{\star}}{F_{u u}^{\star}} \exp [-\rho \tau]\left(x(t+\tau)-x^{\star}\right), \\
\dot{x}(t) & \approx\left(u(t-\tau)-u^{\star}\right)-\gamma\left(x(t)-x^{\star}\right) .
\end{aligned}
$$

Introducing $A=F_{x u}^{\star} / F_{u u}^{\star}$ and $B=F_{x x}^{\star} / F_{u u}^{\star}$, we derive for the characteristic equation

$$
\begin{aligned}
& Q(z)=z^{2}-\rho z-\gamma(\gamma+\rho)-B \exp [-\rho \tau] \\
& \quad+A\{\exp [-z \tau](z-\rho-\gamma)-\exp [(z-\rho) \tau](z+\gamma)\} .
\end{aligned}
$$

$Q(z)$ is a quasi-polynomial, which exhibits, in general, an infinite number of roots with negative real parts and an infinite number of roots with positive real parts, but reduces to a quadratic equation if the cross derivative $F_{x u}^{\star}$ vanishes $(A=0)$. The following proposition elaborates on the properties of the characteristic roots.

PROPOSITION 2 (Roots of the Characteristic Equation). For the roots of the characteristic equation (9) the following statements hold:

(i) The characteristic roots of (9) are symmetric around $\rho / 2$.

(ii) If $F_{x u}\left(x^{\star}, s^{\star}\right)=0$, (9) exhibits only two real roots $z_{1 / 2}$ with $z_{1}<0$ and $z_{2}>\rho$.

(iii) Otherwise, (9) exhibits zero, two, or four real roots, which are either smaller than zero or larger than $\rho$, and an infinite number of conjugate pairs of complex roots with unbounded positive and unbounded negative real parts.

The proof is given in the Appendix.

The general solution of the system of differential-difference equations (8) can be written as infinite sums of exponential functions [Bellman and Cooke (1963); 
Boucekkine et al. (2005)]. Indexing the real roots of the characteristic equation (9) by $r$ and the complex roots by $c$, we obtain ${ }^{5}$

$$
\begin{aligned}
& u(t)=u^{\star}+\sum_{r} u_{r} \exp \left[z_{r} t\right]+\sum_{c} u_{c}^{1} \exp \left[a_{c} t\right] \cos \left[b_{c} t+u_{c}^{2}\right], \\
& x(t)=x^{\star}+\sum_{r} x_{r} \exp \left[z_{r} t\right]+\sum_{c} x_{c}^{1} \exp \left[a_{c} t\right] \cos \left[b_{c} t+x_{c}^{2}\right],
\end{aligned}
$$

where $z_{r}$ is the real root with index $r, a_{c}$ and $b_{c}$ are the real and imaginary parts of the complex root $z_{c}$ with index $c$, and $u_{r}, u_{c}^{1}, u_{c}^{2}, x_{r}, x_{c}^{1}$, and $x_{c}^{2}$ are real constants that have to be determined by the initial and transversality conditions. Utilizing the transversality condition (4c) and the nonnegativity constraints (2b), we can now determine the optimal system dynamics in a neighborhood around the stationary state.

PROPOSITION 3 (Optimal Paths). The general solution of the system of differential-difference equations (8) that is consistent with the transversality condition (4c) and the nonnegativity constraints (2b) is given by

$$
\begin{aligned}
& u(t)=u^{\star}+\sum_{r^{-}} u_{r^{-}} \exp \left[z_{r^{-}} t\right]+\sum_{c_{0}^{-}} u_{c_{0}^{-}}^{1} \exp \left[a_{c_{0}^{-}} t\right] \cos \left[b_{c_{0}^{-}} t+u_{c_{0}^{-}}^{2}\right], \\
& x(t)=x^{\star}+\sum_{r^{-}} x_{r^{-}} \exp \left[z_{r^{-}} t\right]+\sum_{c_{0}^{-}} x_{c_{0}^{-}}^{1} \exp \left[a_{c_{0}^{-}} t\right] \cos \left[b_{c_{0}^{-}} t+x_{c_{0}^{-}}^{2}\right],
\end{aligned}
$$

where all negative real roots are indexed by $r^{-}$and all complex roots with nonpositive real part are indexed by $c_{0}^{-}$.

The proof is given in the Appendix.

Proposition 3 says that the optimal paths either are converging toward the unique stationary state or may exhibit limit cycles if the characteristic equation exhibits purely imaginary roots. ${ }^{6}$ The intuition is that all roots with positive real parts greater than or equal to the discount rate $\rho$ contradict the transversality condition (4c). Furthermore, all complex roots with positive real part are inconsistent with the nonnegativity constraints (2b). As the characteristic equation (9) does not exhibit any real roots in the interval $[0, \rho]$ (see Proposition 2 ), the optimal solution in a neighborhood around the stationary state consists only of exponential functions with negative or vanishing real part. Thus, the optimal solution either converges toward the stationary state or oscillates around it.

However, the qualitative system dynamics depends crucially on the functional form of the felicity function $F$, as the following proposition shows.

PROPOSITION 4 (Time Lags and Cycles). For the optimal path (11a) the following statements hold: 
(i) If $F_{x u}\left(x^{\star}, s^{\star}\right)=0$, the optimal paths (11a) converge monotonically toward the stationary state.

(ii) Otherwise, the optimal path (11a) either converges to the stationary state in an oscillatory manner or exhibits a limit cycle.

Proof. For $F_{x u}\left(x^{\star}, s^{\star}\right)=0$, the characteristic equation (9) only exhibits a negative real root $z_{1}$ smaller than the discount rate $\rho$. Thus, the optimal path reduces to

$$
u(t)=u^{\star}+u_{1} \exp \left[z_{1} t\right], \quad x(t)=x^{\star}+x_{1} \exp \left[z_{1} t\right] .
$$

Otherwise, the optimal path is given by (11), which is a superposition of an infinite number of exponentially damped oscillations. If the characteristic equation (9) exhibits complex roots with vanishing real part, the long-run behavior of the optimal path is given by trigonometric oscillations around the stationary state.

To better understand the system dynamics in the case $F_{x u}\left(x^{\star}, u^{\star}\right)=0$, we consider the special case where the felicity function $F$ is additively separable in both arguments (which guarantees that $F_{x u}=0$ ). Thus, the system of functional differential equations (6) reduces to a system of ordinary first-order differential equations, where the initial stock $x_{0}$ and the initial path $\mu$ translate into the new initial condition $\tilde{x}(0)=\tilde{x}_{0}=\chi(\tau)$, which is given by equation (6c) for $t=\tau$. To see this, we set $F(x(t), u(t))=F_{1}(x(t))+F_{2}(u(t))$ and $\tilde{x}(t)=x(t+\tau)$ and obtain

$$
\begin{aligned}
& \dot{u}(t)=\frac{1}{F_{2}^{\prime \prime}(u(t))}\left\{(\gamma+\rho) F_{2}^{\prime}(u(t))+F_{1}^{\prime}(\tilde{x}(t)) \exp [-\rho \tau]\right\}, \\
& \dot{\tilde{x}}(t)=u(t)-\gamma \tilde{x}(t) .
\end{aligned}
$$

We see that the crucial difference between equation (13a) and the corresponding equation (6a) is that equation (13a) depends only on $u(t)$ and $x(t+\tau)$ and not simultaneously on $u(t), u(t+\tau), u(t-\tau), x(t)$, and $x(t+\tau)$. That is, both the stock and the control are evaluated not at different times but only at one point in time. In addition, the time structures of equations (13a) and (13b) are identical ( $u$ is evaluated at a time that lies $\tau$ earlier than the time at which $x$ is evaluated) and can be reduced to an ordinary differential equation by a simple variable transformation. Thus, the result that for additively separable $F$ the system dynamics is monotonic hinges on two crucial features. On one hand, with $F$ being additively separable, the first and second derivatives depend only on either $x$ or $u$ and the cross derivative $F_{x u}$ vanishes, which leads to the fact that in equation (13a) the same variable is evaluated at only one point in time. On the other hand, the equation of motion of the stock (13b) is of the same structure, as it depends on variables that are only evaluated at one point in time and, in addition, the delay structure is the same as the resulting delay structure in equation (13a). ${ }^{7}$ 
In summary, the introduction of the delay $\tau$ leaves the stability properties of the unique stationary state almost unaltered (if we abstract from the case of limit cycles), but it may change the qualitative system dynamics. If the felicity function is additively separable, the optimal system dynamics shows a monotonic convergence toward the stationary state, as is the case for instantaneous stock accumulation. If, however, the felicity function is not additively separable, the system dynamics exhibits oscillatory behavior.

\section{APPLICATIONS}

Finally, we come back to the examples discussed in Section 2. Recall the neoclassical growth model with the felicity function $F=V(P(k(t))-i(t))$. Assuming that capital needs the time span $\tau$ to become productive and that capital depreciates at the constant rate $\gamma$, the resulting optimal control problem is of the form (1) discussed in Section 3. As the felicity function is never additively separable in the control $i$ and the stock $k$, the optimal path converges in an oscillatory manner toward the stationary state or exhibits limit cycles, as already shown by Rustichini (1989) and Asea and Zak (1999). A special case, the AK growth model, was analyzed in detail by Bambi (2008) and Winkler (2009). They showed that the optimal system dynamics converges in an oscillatory manner toward the longrun balanced growth path or exhibits a limit cycle around it. Furthermore, the oscillatory behavior is the more pronounced the larger is the delay $\tau$.

The situation is more complex in partial equilibrium models, where the felicity function may be either additively separable or not. We further discuss the optimal accumulation of a pollution stock. We introduce a general felicity function of the generic type discussed in Section 3 to address the case where $F$ may not be additively separable, ${ }^{8}$

$$
F=-\left\{\frac{\phi}{2}[\epsilon-e(t)]^{2}-\alpha[\epsilon-e(t)] s(t)+\frac{\beta}{2} s(t)^{2}\right\},
$$

where $\epsilon$ denotes "business-as-usual" emissions and, thus, $\epsilon-e$ can be interpreted as emission abatement. Note that the felicity function is the sum of three terms. The first depends only on emissions $e$ and captures the direct costs of emission abatement. The third depends only on the pollution stock $s$ and denotes the direct damage costs. The second is a feedback term depending on both emissions $e$ and the pollution stock $s$, which implies that the damage due to the pollution stock $s$ decreases with decreasing emissions $e$ (increasing abatement). Assuming that the stock accumulates with a delay $\tau$ and depreciates at a constant rate $\gamma$, i.e., $\dot{s}(t)=e(t-\tau)-\gamma s(t)$, we obtain an optimal control problem of the form (1). ${ }^{9}$

The parameter $\alpha$ indicates the strength of the feedback term. For $\alpha=0$ we obtain an additively separable felicity function $F$. Due to its special form, the optimal path can be derived analytically (see Appendix A.3). In line with Propositions 2 and 4 , the characteristic equation exhibits only one real root smaller than the 

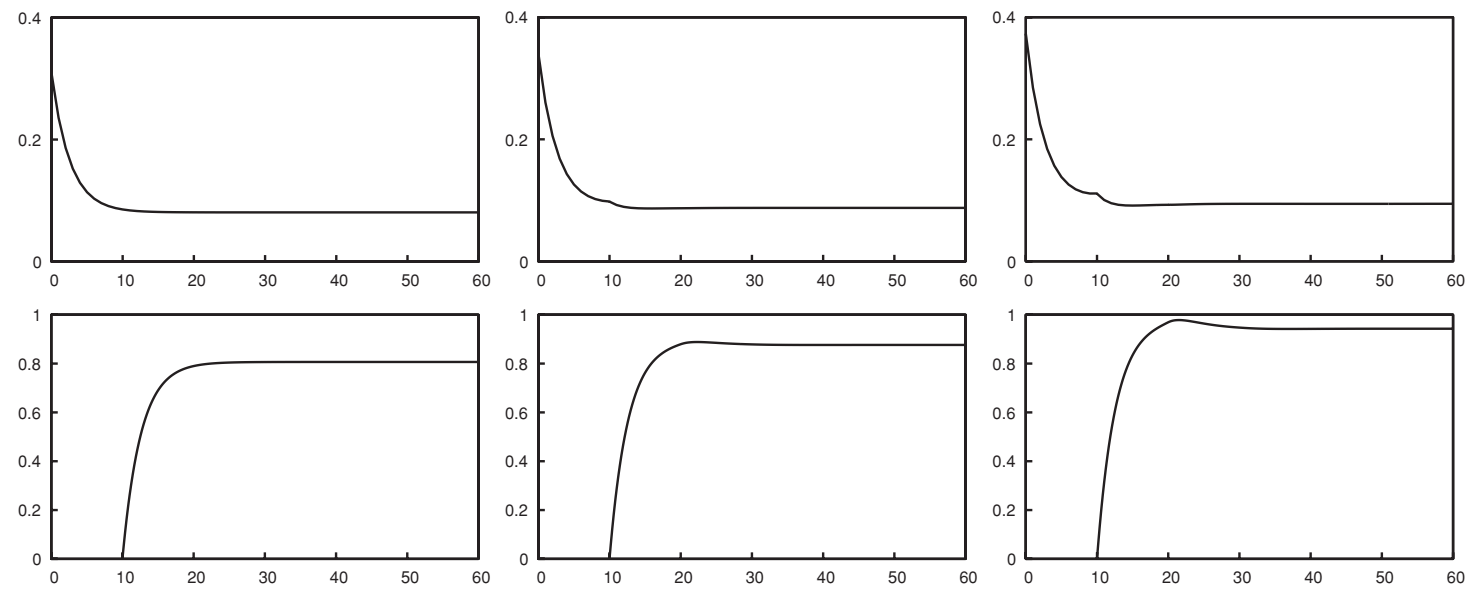

FIGURE 1. Optimal paths for emissions (top) and pollution stocks (bottom) for feedback strengths $\alpha=0$ (left), $\alpha=0.01$ (middle), and $\alpha=0.02$ (right). 
discount rate $\rho$ and, thus, the optimal paths converge monotonically toward the unique stationary state.

For $\alpha>0$ the optimal control problem cannot be solved analytically. As a consequence, we numerically solve the optimal paths of emissions and the pollution stock. ${ }^{10}$ Figure 1 shows the optimal paths for emissions $e$ (top) and the pollution stock $s$ (bottom) for three different values of the feedback strength $\alpha(0,0.01$, and 0.02). Due to the delay $\tau=10$ and the vanishing initial stock $s_{0}=0$ and initial emission path $\mu(t)=0, \forall t \in[-\tau, 0)$, the stock is equal to zero in the time interval $[0, \tau]$. For $\alpha=0$ the optimal paths of emissions $e$ and the pollution stock $s$ converge monotonically to their stationary state values. For positive values of $\alpha$ we observe that the optimal emission path exhibits a kink at $t=\tau=10$. Moreover, the optimal paths of emissions and the pollution stock are nonmonotonic: we observe an "undershooting" of the optimal emission path and a corresponding "overshooting" of the optimal pollution stock. The reason for both effects is due to the combination of the delay and the feedback term. With respect to the former effect, observe that for $t<\tau$ the pollution stock is equal to zero and, therefore, the feedback term vanishes. At $t=\tau$ the pollution stock becomes positive, implying a positive feedback term. Thus, the LHS of the necessary condition (4a) suddenly increases at $t=\tau$ because of the nonsmooth increase of the pollution stock $s$, whereas the RHS (the shadow price of the pollution stock) remains constant. As a consequence, abatement increases (emissions decrease) in a nonsmooth manner. With regard to the second effect, high costs due to a high pollution stock today can be diminished by additional abatement effort today, but this increased abatement effort reduces the stock with a delay. This leads to nonmonotonic optimal paths.

\section{NOTES}

1. Boucekkine et al. (1997, 1998, 2001, 2005) investigate oscillatory behavior in vintage capital models, whereas de la Croix and Licandro (1999) and Boucekkine et al. (2002, 2004) consider age structures in human capital generated by endogenous schooling and retirement decisions. Bambi (2008) and Winkler (2009) analyze time to build in an AK growth framework, whereas d'Albis and Augeraud-Véron (2009) derive the dynamics of a competitive growth model with a continuous-time overlapping-generations structure.

2. See, e.g., Falk and Mendelsohn (1993), Goulder and Mathai (2000), and Moslener and Requate (2007, 2008).

3. Throughout the paper partial derivatives are denoted by subscripts, derivatives with respect to the sole argument are denoted by primes, and derivatives with respect to time are denoted by dots.

4. Functions evaluated at the stationary state $\left(x^{\star}, u^{\star}\right)$ are denoted by a star.

5. As all complex roots come in conjugate pairs, the general solution of the control $u$ is given by $u(t)=u^{\star}+\sum_{r} u_{r} \exp \left[z_{r} t\right]+\sum_{c} \exp \left[a_{c} t\right]\left(U_{c} \exp \left[i b_{c} t\right]+\bar{U}_{c} \exp \left[-i b_{c} t\right]\right)$, where $U_{c}$ is a complex constant and $\bar{U}_{c}$ denotes its complex conjugate. This is equivalent to expression (10a) [Gandolfo (1996, Ch. 14.1.3)]. Of course, the same applies to the general solution of the stock $x$.

6. That is, the optimal paths oscillate around the stationary state without converging toward or diverging from it. Limit cycles in the case of delayed optimal control problems have been discussed by Rustichini (1989) and Asea and Zak (1999), among others. 
7. This implies that the monotonicity result is not valid for arbitrary equations of motion. In fact, the most general form of the equation of motion preserving this characteristic is $\dot{x}=G(u(t-\tau))-H(x(t))$, where $G$ and $H$ are two monotonically increasing functions.

8. The minus sign stems from the fact that total costs are to be minimized, but the generic optimization problem (1) is formulated as a maximization problem.

9. Numerous environmental problems are caused by pollutants that accumulate stocks with a delay to their emissions, such as chlorofluorocarbons (CFCs), which cause the depletion of the stratospheric ozone layer, and nitrate and pesticide runoffs from agricultural cultivation, which accumulates in the groundwater and decreases its drinking water quality. Often such delays are due to a time-consuming transportation process, in which the pollutants travel from the emitting source to the place where they accumulate.

10. The numerical optimization has been carried out with the advanced optimal control software package MUSCOD-II [Diehl et al. (2001); Leineweber et al. (2003)], developed by the Simulation and Optimization Group of the Interdisciplinary Center for Scientific Computing, University of Heidelberg. Details of the numerical optimization method are discussed in Brandt-Pollmann et al. (2008). The time horizon for the numerical optimization has been set to 100 years, and all parameters have been chosen so that the system at time $t=100$ is very close to the stationary state (for a more convenient exposition, the figures just show times up to $t=60$ ). Apart from $\alpha$, the following exogenous parameters and initial values have been used for the numerical optimization: $\phi=0.5, \beta=0.1, \gamma=0.1, \rho=0.03$, $\epsilon=1, \tau=10, s_{0}=0, \mu(t)=0$. The parameter values have been chosen primarily to illustrate clearly the different effects, and do not necessarily reflect the characteristics of real environmental pollution problems.

11. In this case, the principal term would be a term with $y^{2} \exp [2 y]$.

\section{REFERENCES}

Asea, P.K. and P.J. Zak (1999) Time-to-build and cycles. Journal of Economic Dynamics and Control 23, 1155-1175.

Bambi, M. (2008) Endogenous growth and time-to-build: The AK case. Journal of Economic Dynamics and Control 32, 1015-1040.

Bellman, R. and K.L. Cooke (1963) Differential-Difference Equations. New York: Academic Press.

Boucekkine, R., D. de la Croix, and O. Licandro (2002) Vintage human capital, demographic trends, and endogenous growth. Journal of Economic Theory 104, 340-375.

Boucekkine, R., D. de la Croix, and O. Licandro (2004) Modelling vintage structures with DDEs: Principles and applications. Mathematical Population Studies 11, 151-179.

Boucekkine, R., M. Germain, and O. Licandro (1997) Replacement echoes in the vintage capital growth model. Journal of Economic Theory 74, 333-348.

Boucekkine, R., M. Germain, O. Licandro, and A. Magnus (1998) Creative destruction, investment volatility, and the average age of capital. Journal of Economic Growth 3, 361-384.

Boucekkine, R., M. Germain, O. Licandro, and A. Magnus (2001) Numerical solution by iterative methods of a class of vintage capital models. Journal of Economic Dynamics and Control 25, 655-669.

Boucekkine, R., O. Licandro, L.A. Puch, and F. del Rio (2005) Vintage capital and the dynamics of the AK model. Journal of Economic Theory 120, 39-72.

Brandt-Pollmann, U., R. Winkler, U. Moslener, and J. Schlöder (2008) Numerical solution of optimal control problems with constant control delays. Computational Economics 31, 181-206.

d'Albis, H. and E. Augeraud-Véron (2009) Competitive growth in a life-cycle model: Existence and uniqueness. International Economic Review 50, 459-84.

de la Croix, D. and O. Licandro (1999) Life expectancy and endogenous growth. Economics Letters 65, 255-263.

Diehl, M., D.B. Leineweber, and A.A.S. Schäfer (2001) Muscod-II users' manual. Preprint 2001-25, Interdisciplinary Center for Scientific Computing, University of Heidelberg. 
El-Hodiri, M.A., E. Loehman, and A. Whinston (1972) An optimal growth model with time lags. Econometrica 40, 1137-1146.

Falk, I. and R. Mendelsohn (1993) The economics of controlling stock pollutants: An efficient strategy for greenhouse gases. Journal of Environmental Economics and Management 25, 7688.

Gandolfo, G. (1996) Economic Dynamics, 3rd completely revised and enlarged edition. Berlin: Springer.

Goulder, L.H. and K. Mathai (2000) Optimal $\mathrm{CO}_{2}$ abatement in the presence of induced technological change. Journal of Environmental Economics and Management 39, 1-38.

Hayek, F.A. (1941) The Pure Theory of Capital. London: Routledge \& Kegan Paul.

Jevons, W.S. ([1871]1911) The Theory of Political Economy, 4th ed. First published in 1871, second, enlarged edition in 1879. London: Macmillan.

Kalecki, M. (1935) A macroeconomic theory of business cycles. Econometrica 3, 327-344.

Kamien, M.I. and N.L. Schwartz (1992) Dynamic Optimization. The Calculus of Variations and Optimal Control in Economics and Management, 2nd ed. Amsterdam: Elsevier.

Kolmanovskii, V.B. and A.D. Myshkis (1999) Introduction to the Theory and Applications of Functional Differential Equations. Dordrecht, The Netherlands: Kluwer.

Kydland, F.E. and E.C. Prescott (1982) Time to build and aggregate fluctuations. Econometrica 50, $1345-1370$.

Leineweber, D.B., I. Bauer, H.G. Bock, and J.P. Schlöder (2003) An efficient multiple shooting based reduced SQP strategy for large-scale dynamic process optimization: I. Theoretical aspects. Computers and Chemical Engineering 27, 157-166.

Moslener, U. and T. Requate (2007) Optimal abatement in dynamic multi-pollutant problems when pollutants can be complements or substitutes. Journal of Economic Dynamics and Control 31, 2293-2316.

Moslener, U. and T. Requate (2008) The dynamics of optimal abatement strategies for multiple pollutants-An illustration in the greenhouse. Ecological Economics 68, 1521-1534.

Rustichini, A. (1989) Hopf bifurcation for functional differential equations of mixed type. Journal of Dynamics and Differential Equations 1, 145-177.

von Böhm-Bawerk, E. ([1889]1921) Kapital und Kapitalzins. Positive Theorie des Kapitals [Capital and Interest. The Positive Theory of Capital], 4th ed. London: Macmillan.

Winkler, R. (2008) Optimal Control of Pollutants with Delayed Stock Accumulation. Working paper 08/91, CER-ETH - Center of Economic Research at ETH Zurich.

Winkler, R. (2009) Time-to-Build in the AK Growth Model: Short-Run Dynamics and Response to Exogenous Shocks. Mimeo, CER-ETH-Center of Economic Research at ETH Zurich.

\section{APPENDIX}

\section{A.1. PROOF OF PROPOSITION 2}

Proof of (i): The characteristic roots are symmetric around $\rho / 2$; i.e., if $z$ is a characteristic root, then $\rho-z$ is also a characteristic root, as $Q(z)$ is symmetric around $\rho / 2$ [one can easily verify that $Q(z)=Q(\rho-z)]$.

Proof of (ii): For $F_{x u}^{\star}=0$, the characteristic equation (9) reduces to a quadratic equation

$$
Q(z)=z^{2}-\rho z-\gamma(\gamma+\rho)-B \exp [-\rho \tau]
$$


which exhibits the two real solutions

$$
\begin{aligned}
& z_{1}=\frac{\rho}{2}-\sqrt{\left(\frac{\rho}{2}\right)^{2}+\gamma(\gamma+\rho)+B \exp [-\rho \tau]}<0, \\
& z_{2}=\frac{\rho}{2}+\sqrt{\left(\frac{\rho}{2}\right)^{2}+\gamma(\gamma+\rho)+B \exp [-\rho \tau]}>\rho .
\end{aligned}
$$

Proof of (iii): In order to analyze the roots of (9), we write it as the sum of two functions $Q(z)=Q_{1}(z)+Q_{2}(z)$ with

$$
\begin{aligned}
& Q_{1}(z)=z^{2}-\rho z-\gamma(\gamma+\rho)-B \exp [-\rho \tau], \\
& Q_{2}(z)=A\{\exp [-z \tau](z-\rho-\gamma)-\exp [(z-\rho) \tau](z+\gamma)\} .
\end{aligned}
$$

The following properties hold for $Q_{1}$ and $Q_{2}$ :

$Q_{1}^{\prime} \geq 0, \quad Q_{1}^{\prime}=0 \Leftrightarrow x=\frac{\rho}{2}, \quad Q_{1}^{\prime \prime}>0, \quad Q_{1}(0)=Q_{1}(\rho)<0, \quad Q_{1}\left(\frac{\rho}{2}\right)<0$,

$Q_{2}^{\prime} \geq 0, \quad Q_{2}^{\prime}=0 \Leftrightarrow x=\frac{\rho}{2}, \quad Q_{2}^{\prime \prime}<0, \quad Q_{2}(0)=Q_{2}(\rho)<0, \quad Q_{2}\left(\frac{\rho}{2}\right)=0$.

Thus, $Q$ is the sum of a convex and a concave function. $Q$ has no real roots in $[0, \rho]$, as both functions are nonpositive on this interval. Moreover, as $\lim _{z \rightarrow \pm \infty} Q(z)=-\infty$ (the exponential function $Q_{2}$ decreases more strongly than the quadratic function $Q_{1}$ rises), $Q$ exhibits two local maxima and a local minimum at $\rho / 2$. Depending on whether $Q$ is negative, zero, or positive at the loci of the local maxima, $Q$ exhibits zero, two, or four real roots.

To analyze the complex roots of the characteristic equation (9), first note that complex roots come in conjugate pairs; i.e., if $z=a+i b, a, b \in \mathbf{R}$, solves $Q(z)=0$ then $a-i b$ does so, too. Second, we introduce the new variable $y=\tau z$ and multiply $Q$ by $\tau^{2} \exp [y]$,

$$
\begin{aligned}
& Q(y)=\exp [y]\left\{y^{2}-y \rho \tau-\tau^{2}[\gamma(\gamma+\rho)-B \exp [-\rho \tau]]\right\} \\
& \quad+\tau A\{(y-\gamma \tau-\rho \tau)-\exp [2 y-\gamma \tau](y+\gamma \tau)\},
\end{aligned}
$$

in order to apply Theorem 13.1 of Bellman and Cooke (1963, p. 441). As $Q(y)$ has no principal term, i.e., a term where the highest power of $y$ and the highest exponential term appear jointly, ${ }^{11} Q(y)$ has "an unbounded number of zeros with arbitrarily large positive real part" (ibid.). But as the characteristic roots are symmetric around $\rho / 2$, this also implies an unbounded number of roots with arbitrarily large negative real part.

\section{A.2. PROOF OF PROPOSITION 3}

First observe that the transversality conditon (4c) excludes all solutions for the stock $x$ with $z_{r} \geq \rho$ and $a_{c} \geq \rho$. By virtue of the equation of motion (2a), this also excludes all solutions for the control $u$ with $z_{r} \geq \rho$ and $a_{c} \geq \rho$. As there are no real roots in the interval $[0, \rho]$, the optimal solution consists only of exponential functions corresponding to the negative real roots. 
To see that all remaining complex roots with positive real parts contradict the nonnegativity constraint (2b), suppose that $i$ is the index of the complex root with highest real part lower than $\rho$. Thus, there exists a time $T$ for which

$$
\left|u_{i}^{1} \exp \left[a_{i} t\right]\right|>\left|u^{\star}+\sum_{r^{-}} u_{r^{-}} \exp \left[z_{r^{-}} t\right]+\sum_{c, a_{c}<\rho, c \neq i} u_{c}^{1} \exp \left[a_{c} t\right]\right|, \quad \forall t>T .
$$

As $\cos \left[b_{i} t+u_{i}^{2}\right]=-1$ for $b_{i} t+u_{i}^{2}=(2 l+1) \pi, l \in \mathbf{N}_{0}$, the control $u$ is at times negative for $t>T$, which contradicts (2b). The analogous argument holds for the stock $x$.

\section{A.3. DERIVATION OF THE OPTIMAL PATHS FOR AN ADDITIVELY SEPARABLE FELICITY FUNCTION}

Setting $\alpha=0$ and applying Proposition 1 yields the following unique stationary state:

$$
s^{\star}=\frac{\epsilon \phi(\gamma+\rho) \exp [\rho \tau]}{\beta+\phi \gamma(\gamma+\rho) \exp [\rho \tau]}, \quad e^{\star}=\frac{\gamma \epsilon \phi(\gamma+\rho) \exp [\rho \tau]}{\beta+\phi \gamma(\gamma+\rho) \exp [\rho \tau]} .
$$

The optimal path is given by

$$
\begin{aligned}
& \dot{e}(t)=(\gamma+\rho)\left[e(t)-e^{\star}\right]+\frac{\beta}{\phi} \exp [-\rho \tau]\left[\tilde{s}(t)-s^{\star}\right], \\
& \dot{\tilde{s}}(t)=\left[e(t)-e^{\star}\right]-\gamma\left[\tilde{s}(t)-s^{\star}\right]
\end{aligned}
$$

where $\tilde{s}=s(t-\tau)$ and $\tilde{s}(0)=\chi(\tau)=0$. We obtain $A=0$ and for $B=\beta / \phi$. According to Proposition 2, the characteristic equation exhibits a unique real root $z_{1}$ smaller than the discount rate $\rho$,

$$
z_{1}=\frac{\rho}{2}-\sqrt{\left(\frac{\rho}{2}\right)^{2}+\gamma(\gamma+\rho)+\frac{\beta}{\phi} \exp [-\rho \tau]}<0
$$

Then, the solution of the optimal path yields

$$
\begin{aligned}
& e(t)=e^{\star}+s^{\star} \frac{\beta \exp [-\rho \tau]}{(\gamma+\rho)-z_{1}} \exp \left[z_{1} t\right], \quad t \geq 0, \\
& s(t)=s^{\star}\left\{1-\exp \left[z_{1}(t-\tau)\right]\right\}, \quad t \geq \tau, \\
& s(t)=0, \quad t \in[0, \tau] .
\end{aligned}
$$

